was expanded almost four-fold from an original estimated 5000 participants.

To conclude, it is difficult to draw conclusions from this very interesting study as it is not transparently reported. With many unanswered questions, it is too early to change clinical practice. There are two ongoing studies testing similar hypotheses, the BedMed and TIME study. It will be very important to follow the outcome of these trials.

Bo Carlberg - bo.carlberg@umu.se

\section{References}

1. Hermida RC, Crespo JJ, Domínguez-Sardina $M$ et al. Bedtime hypertension treatment improves cardiovascular risk reduction: the Hygia Chronotherapy Trial. Eur Heart J 2019; doi: 10.1093/eurheartj/ehz754
2. Schulz KF, Altman DG, Moher D et al. CONSORT 2010 Statement: Updated Guidelines for Reporting Parallel Group Randomized Trials. Ann Intern Med 2010;152:726-32. doi: 10.1097/AOG.0b013e3181d9d421

\title{
INVITED COMMENT: \\ New NICE Guideline opts for stability
}

\author{
JOHN CHALMERS \\ The George Institute for Global Health \\ The University of NSW, Sydney \\ Australia
}

Following the publication of the SPRINT study in the New Engl J Med in 2015', a number of the major international guidelines have gradually been revised, beginning with those of the American College of Cardiology and American Heart Association (AHA) in $2017^{2}$. These were followed by the guidelines of the European Societies of Cardiology and of Hypertension (ESC/ESH) in $2018^{3}$ and then by the British guidelines for "Hypertension in Adults: diagnosis and management" from the National Institute for Health and Care Excellence ('NICE')'.

One of the attractive features of NICE is that these guidelines are written in plain and simple English and are aimed as much at patients and their families and carers, as they are at hypertension specialists, general practitioners or other health care professionals ${ }^{4}$. Another attractive feature is their relative brevity and simplicity, though that is achieved in part by referring the reader to a variety of separate NICE guidelines such as those on "chronic kidney disease in adults", or "cardiovascular disease" or "alcoholuse disorders" or on "stop smoking interventions and services". On the other hand, these new NICE guidelines do apply to people with type 2 diabetes ${ }^{4}$.

\section{Opportunities for change that were resisted}

These are no major dramatic changes in the updated NICE guidelines though they do refer to the relevant literature, albeit with a conservative bent. The major areas where possible change has been resisted include the following:

\section{Measurement of Blood Pressure}

The 2019 NICE guideline does not consider the possible use of "Unattended Automated Office Blood Pressure Measurement", (AOBPM) the method used in SPRINT \& recommended in the ACC/AHA 2017 guidelines as well as in the 2016 Canadian Guidelines 5 , on the grounds that this method of measuring BP would be difficult to translate into UK clinical practice. They prefer the more traditional use of automated devices or of manual measurement using direct auscultation over the brachial artery ${ }^{4}$. That may well be true but a recommendation from NICE in 2019 encouraging the use of AOBPM might have provided valuable leadership. It is interesting to note that in a recent survey of patients with 
resistant hypertension across 76 specialist centres from 6 major regions, completed in August 2016, the preferred method of BP measurement reported was AOBPM in $97 \%$ of patients in Australia and $87 \%$ in North America (USA \& Canada).

\section{Setting of BP thresholds and targets for treatment}

The 2019 NICE guideline retains a BP of 140/90 $\mathrm{mmHg}$ as the threshold for the diagnosis of hypertension or high BP and as the target for the treatment of high BP. For out-of-office BP these readings are reduced to $135 / 85 \mathrm{mmHg}$, for both daytime Ambulatory Blood Pressure Monitoring (ABPM) and Home Blood Pressure Monitoring (HBPM).

While the authors recognise that there is evidence from the SPRINT trial that lower BP thresholds and targets do confer significant benefits, they point out that this is accompanied by significant harms. They also have concerns that many aspects of the trial, which was conducted in the USA, including the patient populations, and the method for measurement of BP (AOBPM) "made the evidence difficult to interpret and use to inform the recommendations". ${ }^{4}$ In part this might be because the focus of the NICE Guideline on Hypertension in Adults is primarily on primary prevention and treatment, which also may explain how they can quite readily ignore or outweigh the evidence of meta-analyses such as that by Ettehad et al $^{7}$ which may be more relevant to one of the other NICE Guidelines, such as the one on "cardiovascular disease".

\section{Starting antihypertensive drug treatment with monotherapy}

NICE 2019 continues to recommend starting with monotherapy in part because the results of the Pathway-1 trial, suggesting dual therapy could help achieve BP control more rapidly, were excluded, because the outcome was a surrogate outcome, and in part because the other evidence in favour of dual therapy is based on improved adherence, not on improved efficacy. ${ }^{8,9}$

\section{Changes recommended by the $\mathbf{2 0 1 9}$ Guideline}

\section{A bouquet for Including Diabetes}

The new NICE Guideline is the first to include people with diabetes, and furthermore their threshold and target BPs are the same as those for people without diabetes. This helps to simplify many issues for the practicing doctor.

\section{New cardiovascular risk threshold of $10 \%$ for treatment in stage 1 hypertension}

Having resisted any temptation to lower the BP threshold for treatment, the authors of the 2019 NICE Guideline do lower the 10-year cardiovascular risk threshold for initiation of treatment in people with stage 1 hypertension below 80 years, from the previous 20\%, to the new level of $10 \%$ ! This recommendation was supported by a health economic analysis which confirmed that treatment was cost effective at the 10\% threshold in people aged 60 years. Given the increasing emphasis on total cardiovascular risk, in preference to blood pressure alone, this makes good sense.

\section{New recommendations for taking BP in the two arms}

The 2019 NICE Guideline continues to recommend BP should be measured in both arms at the time of diagnosis, but recommends a difference of 15 rather than the previous $20 \mathrm{mmHg}$ should be regarded as significant, and subsequent readings should be taken from the arm with the highest reading, with the patient informed which that is. This was in line with recent evidence that quite small differences in arm BP are associated with increased risk of cardiovascular events.

\section{Conclusions}

I have long been a believer in the benefits of lowering blood pressure, and was a little disappointed that NICE 2019 chose not to act on the evidence of the SPRINT trial and of numerous meta-analyses to lower the BP threshold and target for various groups, but I did appreciate the consolation prize, in that the 10 year cardiovascular risk threshold for treatment in people below 80 with stage 1 hypertension was lowered to $10 \%$ from the previous $20 \%$. As a long-time advocate of combination therapy for the treatment of hypertension, and a firm believer in the merits of using single pill combinations for the initiation of therapy, I was again disappointed. I was however delighted to see people with type 2 diabetes included in the NICE 2019 Hypertension Guideline, with the same threshold and target BP as people without diabetes. And I did appreciate the way NICE spoke to the reader, and explained its point of view - a real lesson for all the other guidelines which are strictly written for the expert and require that person at their sharpest and fit enough to run a marathon! Finally, it seems likely 
that for many doctors and their patients in the UK - and that is for whom these guidelines are meant - they will be welcomed as "steady as she goes", with a few sensible changes and a simple regime to follow, with a common threshold and target BP, but nothing too radical. Let's hope that significantly more people with high blood pressure will actually take their medication and achieve that target clinic blood pressure of $<140 \mathrm{mmHg}$. That would be progress!

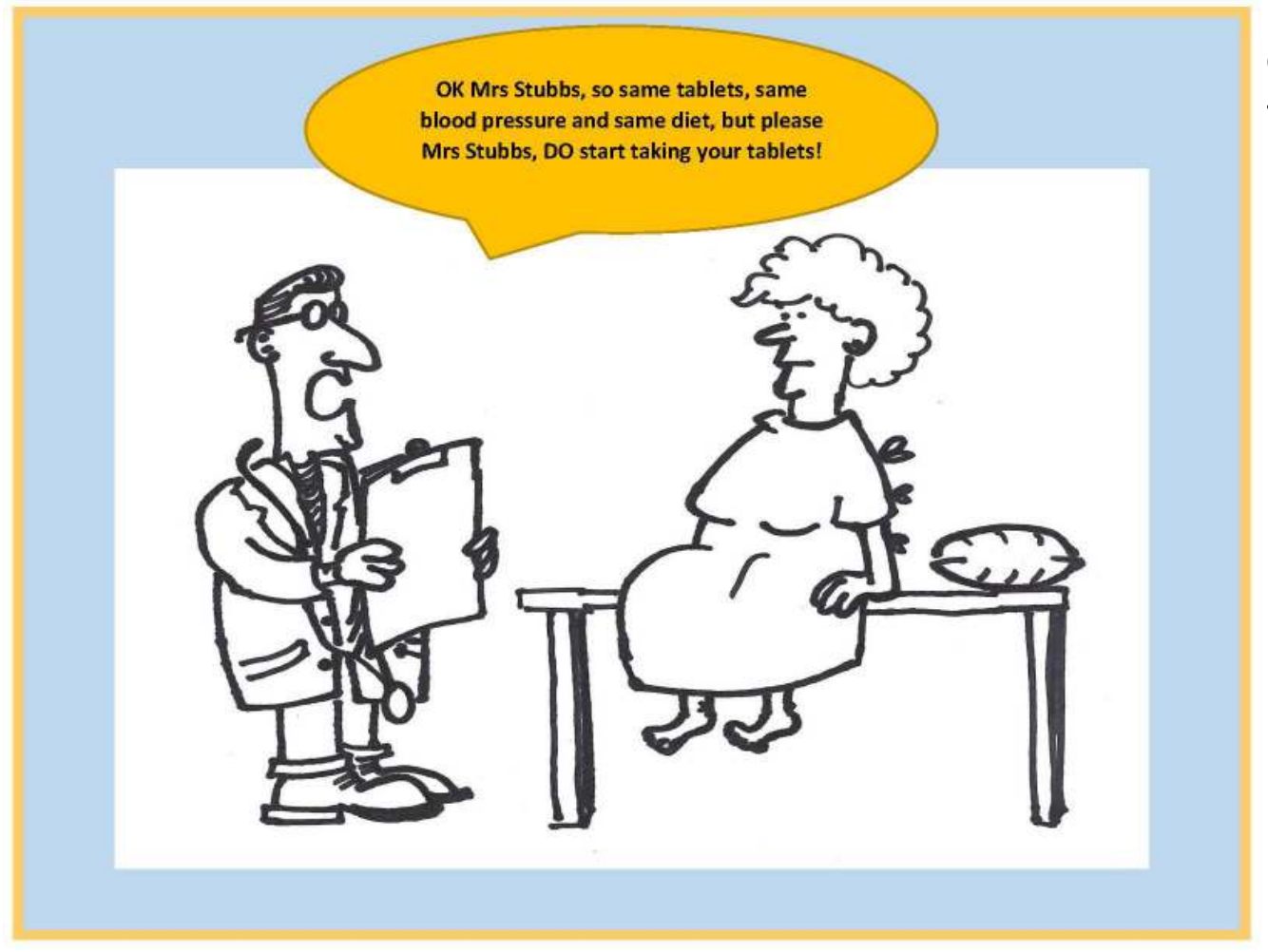

Cartoon by Jordi Carreras - Sydney, Australia

John Chalmers - chalmers@georgeinstitute.org.au

\section{References}

1. Wright JT, Williamson JD, Whelton PK et al for the SPRINT Research Group. A randomised trial of intensive versus standard blood pressure control. N Engl J Med 2015;373:21032116 doi: 10.1056/NEJMoa1511939

2.Whelton PK, Carey RM, Aronow WS et al (2017) ACC/ AHA/AAPA/ABC/ACPM/AGS/APhA/ASH/ASPC/NMA/PCNA Guidelines for the prevention, detection, evaluation and management of high blood pressure in adults: a report of the American College of Cardiology/American Heart Association Task Force on Clinical Practice Guidelines. J Am Coll Cardiol. 2018;71:e127-e248 doi: 10.1016/j.jacc.2017.11.006 [published Online First 2017/11/18]

3.Williams B, Mancia G, Spiering W et al. 2018 ESC/ESH Guidelines for the management of arterial hypertension. Journal of Hypertension 2018; 36:1953-2041 doi: 10.1097/ HJH.0000000000001940

4.The National Institute for Health and Care Excellence. Hypertension in Adults: diagnosis and management. NICE guideline 136.London.National Institute for Health and Care Excellence 2019. https//www.nice.org.uk.guidance/ng136
5.Leung A, Nerenberg K, Daskalopoulou SS et al, Hypertension Canada's 2016 Canadian Hypertension Education Program Guidelines for Blood Pressure Measurement, Diagnosis, assessment of Risk, Prevention, and Treatment of Hypertension. Canadian Journal of Cardiology 2016; 32:569-588 doi: 10.1016/j.cjca.2016.02.066

6.Carcel C, Neal B, Oparil S et al. Clinical characteristics, antihypertensive medication use and blood pressure control among patients with treatment-resistant hypertension: the Survey of Patlents with treatment Reslstant hyperTension study. J Hypertens 2019;37:2216-2224 doi: 10.1097/ HJH.0000000000002184

7.Ettehad D, Emdin C, Kiran A et al. Blood Pressure lowering for prevention of cardiovascular disease and death: a systematic review and meta-analysis. The Lancet 2016; 387:957-67 doi: 10.1016/S0140-6736(15)01225-8

8. Gupta P, Patel P, Strauch B et al. Biochemical screening for non-adherence is associated with blood pressure reduction and improvement in adherence. Hypertension 2017; 701:1042-48 doi: 10.1161/HYPERTENSIONAHA.117.09631

9. Corrao P, Parodi A, Nicotra F et al. Better compliance to antihypertensive medications reduces cardiovascular risk. J Hypertension 2011; 29:610-618 doi: 10.1097/ HJH.0b013e328342ca97 